\title{
CONSTRUCTING KNOWLEDGE DATABASE IN DECISION MAKING
}

The paper describes solving problem of constructing knowledge database of a decision making in process safety. It provides analyses of the requirements as well the analyses of the system incidents caused by specification, design and the implementation of the project. Main focus of this scientific paper is highlighted on practical stability problem and conditions for optimal performance of safe fault-tolerant controllers, I/O, engineering and pressure transmitters. Algorithm of decision making in process safety is developed and the system has been realized taking into account C\# approach in Windows environment.

Key words: decision making, safety, process

\section{Introduction}

One of the most important tasks in the safety engineering lies in the construction of a knowledge database of decision support for the chemical plants, and that way to ensure optimal conditions, improve quality and boost efficiency. Methods of analysis of control systems and simulation methods, which are used for observing dynamic behavior of linear systems with time delay, and distributed parameter systems, based on linear algebra, operation calculus, functional analysis, integral differential equations and linear matrix non-equations showed long ago that modern electronic components can be used to achieve more consistent quality at lower costs in safety engineering. The main idea to do so is that the quality service is maintained and controlled. Applying the Fuzzy theory in decision making has given very good results, and provided a flexible framework and over the years numerous mathematical models have been developed.

There are two basic problems to solve in decision making situations: obtaining alternative, and achieving consensus about solution from group of experts. First problem takes into account individual information which existed in collective information units. The latter usually means an agreement of all individual opinions. Usually two approaches are considered for developing a choice process in solving decision making problems: a direct approach where solution is derived on the basis of the individual relations and as well indirect approach where solution is based on a collective preference relation. In safe engineering using of the PLC has shown technical and economic benefits over hard-wired components. A main problem in process engineering is practical stability of the system. The chosen system should be stable in required period of time, and this important task is obtained by using practical stability theory for distributed parameter systems. Most systems in chemical engineering as chemical plants for instance, are described by partial different equations and they belong to a group of distributed parameter systems.

\section{Practical Stability}

During the process of analysis and synthesis of control systems a fundamental problem is stability. It is a well-known fact, that we can share stability definitions to Ljapunov and non-Ljapunov concepts that have arisen from various engineering needs. The most often case for consideration of control systems is Ljapunov approach, where the system behavior is considered on infinite interval which in real cases has only academic importance. From strictly engineering point of view it is very important to know the boundaries of the system trajectory in state space. These practical technical needs are responsible for non-Ljapunov definitions, and among them is extremely important behavior on finite time interval- practical stability. Taking into account that the system can be stable in the classical way lacking appropriate quality of dynamic behavior, and because of that it is not applicable, it is important to take the system in consideration in relation with sets of permitted states in phase space which are defined for such a problem. In theory of control systems there are demands for stability on finite time interval that for strictly engineering point of view has tremendous importance. The basic difference between Ljapunov and practical stability is set of initial states of system $\left(S_{\alpha}\right)$ [2], and set of permitted disturbance $\left(S_{\varepsilon}\right)$ in state space, for every opened set $S_{\beta}$ permitted states the equilibrium point of that system will be totally stable,

\footnotetext{
* Dj. N. Dihovicni

Technical University, Belgrade 11000, Serbia; E-mail: ddihovic@Eunet.rs
} 
instead the principle of practical stability where are sets $\left(S_{\alpha}, S_{\varepsilon}\right)$ and set $S_{\beta}$ which is closed, determined and known in advance.

Taking into account the principle of practical stability, the following conditions must be satisfied:

- determine set $S_{\beta^{-}}$find the borders for system motion

- determine set $S_{\varepsilon^{-}}$find maximum amplitudes of possible disturbance

- determine set $S_{\alpha}$ of all initial state values.

In case that these conditions are regularly determined it is possible to analyse the system stability from practical stability view of point.

\section{Definitions and Conditions of Practical Stability}

Let us consider first order hyperbolic distributed parameter system, which is decribed by the following state- space equation:

$$
\frac{\partial \underline{x}(t, z)}{\partial t}=A_{0} \cdot \underline{x}(t, z)+A_{1} \frac{\partial \underline{x}}{\partial z}
$$

with appropriate function of initial state

$$
\begin{gathered}
\underline{x}_{0}(t, z)=\underline{\psi}_{x}(t, z) \\
0 \leq t \leq \tau, 0 \leq z \leq \zeta
\end{gathered},
$$

where $x(t, z)$ is n-component real vector of the system state, $A$ is the matrix appropriate dimension, $t$ is time and $z$ is space coordinate.

Definition 1: The distributed parameter system described by equation (1) that satisfies initial condition (2) is stable on finite time interval in relation to $[\xi(t, z), \beta, T, Z]$ if and only if:

$$
\begin{gathered}
\underline{\psi}_{x}^{T}(t, z) \cdot \underline{\psi}_{x}(t, z)<\zeta(t, z) \\
\quad \forall t \in[0, T], \forall z \in[0, \zeta]
\end{gathered}
$$

then it follows

$$
\begin{gathered}
\left.\underline{x}^{T}(t, z)\right) \cdot \underline{x}(t, z)<\beta \\
\forall t \in[0, T] \forall z \in[0, Z]
\end{gathered}
$$

where $\xi(t, z)$ is scalar function with feature $0<\xi(t, z) \leq \alpha, 0 \leq t \leq$ $\leq \tau, 0 \leq z \leq \zeta$ where $\alpha$ is real number, $\beta \in R$ and $\beta>\alpha$.

Let us calculate the fundamental matrix for this class of system:

$$
\frac{d \Phi(s, \sigma)}{d \sigma}=A_{1} \cdot(s I-A) \cdot \Phi(s, \sigma),
$$

where after double Laplace transformation the fundamental matrix is given by the equation, Dihovicni et al. [4]:

$$
\begin{gathered}
\Phi(t, z)=\exp (A \cdot t \cdot z), \\
\text { where } A=\frac{I-A_{0} \cdot A_{1}}{A_{1}} .
\end{gathered}
$$

Theorem1: The distributed parameter system described by equation (1) that satisfies internal condition (2) is stable on finite time interval in relation to $[\xi(t, z), \beta, T, Z]$ if and only if: $e^{2 \mu(A) \cdot t \cdot z}<\frac{\beta}{\alpha}$

Proof: Solution of equation (1) with initial condition (2) is possible to describe as:

$\underline{x}(t, z)=\Phi(t, z) \cdot \underline{\psi}(0,0)$.

By using the above equation it follows:

$x^{T}(t, z) \cdot \underline{x}^{T}(t, z)=\left\lfloor\underline{\psi}_{x}^{T}(0,0) \cdot \Phi(t, z)\right] \cdot\left[\underline{\psi}_{x}^{T}(0,0) \cdot \Phi(t, z)\right] \cdot(9)$

By using well-known ineqality

$$
\|\Phi(t, z)\|=\|\exp [A \cdot t \cdot z]\| \leq \exp \{\mu(A) \cdot t \cdot z\}
$$

and taking into account that:

$$
\begin{aligned}
& \underline{\psi}_{x}^{T}(0,0) \cdot \underline{\psi}_{x}(0,0)<\alpha \\
& \left(\left\|\underline{\psi}_{x}^{T}(0,0)\right\|=\left\|\underline{\psi}_{x}^{T}(0,0)\right\|<\alpha\right)
\end{aligned}
$$

then it follows:

$$
\underline{x}^{T}(t, z) \cdot \underline{x}(t, z) \leq e^{2 \mu(A \cdot t z)} \cdot \alpha .
$$

Applying the basic condition of theorem 1 by using equation (7) to further inequality we obtain Dihovicni et al. [4]:

$$
\underline{x}^{T}(t, z) \cdot \underline{x}(t, z)<\left(\frac{\beta}{\alpha}\right) \cdot \alpha<\beta .
$$

Theorem 2: The distributed parameter system described by equation (1) that satisfied initial condition (2) is stable on finite time interval in relation to $[\xi(t, z), \beta, T, Z]$ if and only if:

$$
\begin{aligned}
& e^{\mu(A) \cdot t z}<\frac{\sqrt{\beta / \alpha}}{1+\tau \cdot \zeta\|A\|} \\
& \forall t \in[0, \tau] \forall z \in[0, S]
\end{aligned}
$$

The proof of this theorem is given in Dihovicni et al. [3].

Let $|\underline{x}|_{(\cdot)}$ be any vector norm and any matrix norm $\|\cdot\|_{2}$ which originated from this vector. Following expresions are used:

$$
|\underline{x}|_{2}=\left(\underline{x}^{T} \cdot \underline{x}\right)^{1 / 2} \text { and }\|\cdot\|_{2}=\lambda_{\max }^{1 / 2}\left(A^{*} \cdot A\right),
$$

where * and $T$ are transpose-conjugate and transport matrixes.

It is important to define the matrix measure as:

$$
\mu(A)=\lim _{\varepsilon \rightarrow 0} \frac{\|1+\varepsilon \cdot A\|-1}{\varepsilon} .
$$

The matrix measure $\mu$ may be defined in three different forms according to the norm which is used: 


$$
\begin{aligned}
& \mu_{1}(A)=\max \left(\operatorname{Re}\left(a_{k k}\right)+\sum_{i=1, i \neq k}^{n}\left|a_{i k}\right|\right) \\
& \mu_{2}(A)=\frac{1}{2} \max \lambda_{i}\left(A^{T}+A\right) . \\
& \mu_{\infty}(A)=\max \left(\operatorname{Re}\left(a_{i i}\right)+\sum_{k=1}^{n}\left|a_{k i}\right|\right)
\end{aligned}
$$

Definition 2: The distributed parameter system described by equation (1) that satisfies initial condition (2) is stable on finite time interval in relation to $[\xi(t, z), \beta, T, Z]$ if and only if, Dihovicni et al. [3]:

$$
\left|\underline{\psi}_{x}(t, z)\right|_{2}<\xi(t, z)
$$

then follows

$$
|\underline{x}(t)|_{2}<\beta,
$$

where $\xi(t, z)$ is scalar function with feature $0<\xi(t, z) \leq \alpha, 0 \leq t \leq$ $\leq \tau, 0 \leq z \leq \zeta) \alpha$ is real number, $\beta \in \mathrm{R}$ and $\beta>\alpha$.

Theorem 3: The distributed parameter system described by equation (1) that satisfies initial condition (2) is stable on finite time interval in relation to $[\alpha, \beta, T, Z]$ if and only if:

$$
\begin{aligned}
& e^{\mu_{2}(A) \cdot t z}<\frac{\sqrt{\beta / \alpha}}{1+\mu^{-1}{ }_{2}(A)} . \\
& \forall t \in[0, T] \forall z \in[0, Z]
\end{aligned}
$$

Proof: Solution of equation (1) with initial condition (2) is possible to describe by using fundamental matrix as:

$$
\underline{x}(t, z)=\Phi(t, z) \cdot \underline{\psi}_{x}(0,0) .
$$

By using the norms of left and right side of the equation (20) it follows:

$$
\underline{x}^{T}(t, z) \cdot \underline{x}(t, z) \leq e^{2 \mu(A+z)} \cdot \alpha
$$

and by using well-known inequality

$$
\begin{aligned}
& \|\exp (A \cdot t \cdot z)\|_{2} \leq \exp \{\mu(A \cdot t \cdot z)\} \\
& t \geq 0, \quad z \geq 0
\end{aligned}
$$

it follows:

$$
|\underline{x}(t, z)|_{2} \leq e^{\mu_{2}(A) t \cdot z}\left|\underline{\psi}_{x}(0,0)\right|_{2}
$$

and by using equation (17) it is obtained:

$$
|\underline{x}(t, z)|_{2} \leq e^{\mu_{2}(A) \cdot t \cdot z}
$$

so finally it is obtained:

$$
|\underline{x}(t, z)|_{2} \leq \alpha \cdot e^{\mu_{2}(A) \cdot t z}\left\{1+\mu_{2}^{-1}(A)\right\} .
$$

Applying the basic condition of theorem 3 by using equation (19) it is obtained:

$$
\begin{aligned}
& |\underline{x}(t)|_{2}<\beta \\
& \forall t \in[0, T], \forall z \in[0, Z] .
\end{aligned}
$$

Theorem 4: The distributed parameter system described by equation (1) that satisfies initial condition (2) is stable on finite time interval in relation to $[\alpha, \beta, T, Z]$, if and only if Dihovicni et al. [3]:

$$
\begin{aligned}
& e^{\mu(A \cdot t z)}<\frac{\beta}{\alpha} . \\
& \forall t \in[0, T], \forall z \in[0, Z] .
\end{aligned}
$$

Theorem 5: The distributed parameter system described by equation (1) that satisfies initial condition (2) is stable on finite time interval in relation to $\left[t_{0}, J, \alpha, \beta, Z\right]$, if and only if:

$$
\begin{aligned}
& {\left[1+\left(t-t_{0}\right) \cdot \sigma_{\max }\right]^{2} \cdot e^{2\left(t-t_{0}\right) z \cdot \sigma_{\max }}<\frac{\beta}{\alpha},} \\
& \forall t \in[0, T], \forall z \in[0, Z] .
\end{aligned}
$$

where $\sigma_{\max }$ represents the maximum singular value of matrix. The proof of this theorem is given in Dihovicni et al. [4].

\section{Practical Part of Realization}

There are few well known stages in developing computer decision support systems based on knowledge which include choosing suitable mathematical tools, formalization of the subject area [4], and development of the corresponding software. In the first phase the problem lies in making the right diagnosis and in analyses of the requirements and as well the analyses of the system incidents caused by specification, design and the implementation of the project. The problem of diagnostics may be stated such as finite number of subsets Herrera et al. [5], or classical investigation methods should be applied Thayese et al. [6].

The system architecture consists of the following modules:

- Stability checking module. This module is designed as a program for checking the practical stability of the system. If the system passes this check it goes further to other modules.

- Analysis module of safe fault-tolerant controllers, $\mathrm{I} / \mathrm{O}$, engineering and pressure transmitters.

- Diagnostics module.

- Knowledge Module of all possible situations and impacts to chemical plants.

- Optimal solution- decision making module.

- Presentation module.

For system realization an object oriented programming approach has been used, and the program has been developed 
using the $\mathrm{C} \#$ language. Each module has a supportive library and the logical structure is based on the classes, which are described below for ustration.

- Main classes are:

- Analyses group whose primary task is to collect necessary facts about the system.

- Practical stability group which determines whether the system is stable or not. If the system is unstable in view of practical stability, then it is automatically rejected.

- Diagnosis group describes all possible casualities for not required results or potencial casualities for not optimal costs.

- Performance group is used for the optimal performance.

- Cost group is used for the optimal cost effect.

- Decision making algorithm for optimal performance and cost consists of two phases:

- Phase $\mathbf{1}$ is used for input Analyses class, Practical stability class and diagnosis class.
- Phase 2 is used for output Performance and Cost group.

\section{Conclusion}

By analysing process systems from safety and optimal cost perspective, it is important to recognize which systems are not stable in real conditions. From engineering state of view we are interested in such systems which are stable in finite periods of time; so our first concern should be to maintain stable and safe systems. Our knowledge database is created in DB2 and it involves all possible reasons for non adequate performance. Key modules for obtaining the best performance, safety and low costs are a good base for the program support in $\mathrm{C} \#$ programming language and the UML representation.

\section{References}

[1] BERGMANS, J., GUTNIKOV, S., KRASNOPROSHIN, V., POPOK, S., VISSIA, H.: Computer-Base Support to Decision-Making in Orthopaedics, Int'l Conference on Intelligent Technologies in Human Related Sciences, Vol. 2: Leon, 1996, pp. 217-223.

[2] DIHOVICNI, N. DJ., NEDIC, N.: Stability of Distributed Parameter Systems on Finite Space Interval, 32-end Yupiter Conference, Zlatibor, 2006, pp. 306-312.

[3] DIHOVICNI, N.DJ., NEDIC, N.: Stability of Distributed Parameter Systems on Finite Space Interval Described by Cubic Equations, 32-end Yupiter Conference, Zlatibor, 2006, pp. 321-325.

[4] DIHOVICNI, N.DJ., NEDIC, N.: Practical Stability of Linear Systems with Delay in State, AMSE, Association for the Advancement of Modelling \& Simulation Techniques in Enterprises, Tassin La-Demi-Lune, Vol. 62, No. 2, 2007, pp. 98-104.

[5] HERRERA, F., HERRERA-VIEDMA, E., LOPEZ, E.: Computer-Base Support to Decision-Making in Orthopaedics, Int'1 Conference on Intelligent Technologies in Human related Sciences, Vol. 2, Leon, 1996, pp. 205-213.

[6] THAYSE, A., GRIBONT, P.: Approche Logique de l'Inteligence Artificielle l De la Logique, Bordas, 1997. 